\title{
The Role of Chain Length in Nonergodicity Factor and Fragility of Polymers
}

\author{
Cécile Dalle-Ferrier, ${ }^{\dagger}$ Kristine Niss, ${ }^{*}$ Alexei P. Sokolov, ${ }^{\S}$ Bernhard Frick, ${ }^{\S}$ Jorge Serrano,, and \\ Christiane Alba-Simionesco $*, \perp$
}

${ }^{\dagger}$ Laboratoire de Chimie Physique, Bâtiment 349, Université Paris-Sud, 91405 Orsay, France. Current address: Soft Matter group, IPkM, Heinrich Heine Universität, Düsseldorf, Germany, ${ }^{*}$ Laboratoire de Chimie Physique, Bâtiment 349, Université Paris-Sud, 91405 Orsay, France. Current address: Glass and Time, IMFUFA, Department of Sciences, Building 27 Roskilde University RUC Postbox 260 DK-4000 Roskilde, Denmark, ${ }^{\S}$ Chemical Sciences Division at ORNL, Oak Ridge; and Department of Chemistry, University of Tennessee, Knoxville, Tennessee 37996, United States, ${ }^{\circledR}$ Institut Laue Langevin, Grenoble, France,

"ICREA-Departament de Física Aplicada, EPSC, Universitat Politècnica de Catalunya, Esteve Terradas 15, 08860 Castelldefels, Spain, and ${ }^{\perp}$ Laboratoire de Chimie Physique, Bâtiment 349, Université Paris-Sud, 91405 Orsay, France. Current address: Laboratoire Léon Brillouin, CEA-CNRS, Saclay, France

Received July 19, 2010; Revised Manuscript Received September 16, 2010

\begin{abstract}
The mechanism that leads to different fragility values upon approaching the glass transition remains a topic of active discussion. Many researchers are trying to find an answer in the properties of the frozen glassy state. Following this approach, we focus here on a previously proposed relationship between the fragility of glass-formers and their nonergodicity factor, determined by inelastic X-ray scattering (IXS) in the glass. We extend this molecular liquid study to two model polymers- polystyrene (PS) and polyisobutylene (PIB)-for which we change the molecular weight. Polymers offer the opportunity to change the fragility without altering the chemical structure, just by changing the chain length. Thus, we specifically chose PS and PIB because they exhibit opposite dependences of fragility with molecular weight. Our analysis for these two polymers reveals no unique correlation between the fragility and the nonergodicity parameter. Even after a recently suggested correction for a possible contribution of the $\beta$ relaxation, the correlation is not restored. We discuss possible causes for the failure of the "fragility-nonergodicity factor" correlation, emphasizing the features that are specific to polymers. We speculate that polymers might have specific contributions to fragility related to the chain connectivity that are absent in nonpolymeric systems.
\end{abstract}

\section{Introduction}

The mechanism of the steep rise of the structural relaxation time $\tau_{\alpha}$ upon approaching the glass transition temperature $T_{\mathrm{g}}$ remains a controversial topic. The steepness is usually characterized by the so-called isobaric fragility index, defined as ${ }^{1}$

$$
m_{P}=\left(\frac{\partial \log \left(\tau_{\alpha}\right)}{\partial T_{\mathrm{g}} / T}\right)_{P}
$$

Fragility is usually defined at a constant pressure (mostly at ambient $P$ ) and characterizes the deviation of the temperature dependence of $\tau_{\alpha}$ from a simple Arrhenius behavior. The liquids that exhibit almost Arrhenius temperature dependence are called "strong" ( $\left.m_{P} \sim 20-30\right)$, and the liquids with strongly nonArrhenius temperature dependence of $\tau_{\alpha}\left(m_{P} \sim 80-150\right)$ are called "fragile". The classification of liquids according to their fragility plays a major role in attempts to understand the universal slowing down and the freezing of the structural relaxation (i.e., the glass formation). Many theoretical approaches have been proposed to describe the glass transition phenomenon, but none so far was able to describe consistently all the known experimental results. ${ }^{2-10}$ The absence of the winning theoretical concept leads to the very rich phenomenology developed in the

\footnotetext{
*To whom correspondence should be addressed. E-mail: christiane. alba-simionesco@cea.fr.
}

field. In many cases it is based on correlations between the fragility and some other property of the material. It is expected that these correlations could help in identifying the factors that govern the sharp slowing down of the structural relaxation and in disentangling universal from system specific behaviors.

In most of these attempts, the fragility was modified by changing the chemical nature of the liquids. Liquids with different van der Waals, hydrogen- and covalent-bonded interactions, ionic liquids, and polymers were compared. However, this approach, although very useful, often gives rise to large spread of the data and controversies among authors due to specific behaviors related to some details of the intermolecular interactions of each liquid. It becomes therefore interesting to study systems with the fewest possible chemical differences and manipulate their fragility via alternative control parameters such as the molecular weight (chain length) in the case of polymers, or pressure. ${ }^{11-13}$ Polymers have significant differences with other glass-forming materials: while for most of nonpolymeric systems fragility does not go above $\sim 100$, there are many polymers with $m_{P} \sim 140-150$ and higher. ${ }^{14,15}$ Consistently, oligomers (short polymer chains) have fragilities comparable to small molecular van der Waals liquids. However, with increase in the chain length, the fragility might increase sharply, or stay almost constant, or even decrease. ${ }^{16}$ This variation in fragility opens an interesting route for analysis of correlation of $m_{P}$ to other materials properties without altering chemistry and intermolecular interactions of the material.

Published on Web 10/08/2010

pubs.acs.org/Macromolecules 
The idea to correlate fragility, i.e., how rapid a liquid arrests or forms a glass when approaching $T_{\mathrm{g}}$, to some properties of the glass itself, is based on the assumption that the final frozen state, i.e., the glass, carries some memory of how the system fell out of equilibrium. On the basis of striking empirical results, several attempts have been made to relate specific glass properties such as the Boson peak, ${ }^{12-17}$ mean-squared displacement, ${ }^{18}$ Poisson ratio, ${ }^{19}$ broadening of acoustic modes, ${ }^{20}$ or elastic shear modulus $^{21}$ to the fragility index $m_{P}$.

In 2003, Scopigno et al. ${ }^{22}$ suggested a correlation between the isobaric fragility index $m_{P}$ measured above $T_{\mathrm{g}}$ and a parameter $\alpha$, defined as the slope of the inverse nonergodicity factor $f_{Q}$ (obtained by inelastic X-ray scattering (IXS) below $T_{\mathrm{g}}$ ), versus temperature $T$ scaled by $T_{\mathrm{g}}$ :

$$
f_{Q}(T)=\frac{1}{1+\alpha \frac{T}{T_{\mathrm{g}}}}
$$

They found empirically a proportionality between $\alpha$ and fragility when comparing about 10 different low-molecular-weight substances: $\alpha=(135 \pm 10) m_{P}$, low $\alpha$ values corresponding to less fragile systems. This correlation would imply an intimate link between the vibrational properties, the amplitude of the frozen structural fluctuations, and the diffusive properties related to the barrier height that must be overcome in order to achieve the viscous flow. An extension of this work for polymers was proposed by Buchenau et al. ${ }^{23}$ using the long wavelength limit of the nonergodicity factor (i.e., the isothermal compressibility) to estimate $\alpha$. In this case, the correlation with the fragility was not as obvious, and the general trend was not observed for polymers with high fragility.

In order to extend the investigation of this correlation, some of the current authors ${ }^{24}$ have previously reported IXS experiments in which the fragility was changed by applying pressure. This study, which included various molecular liquids with very high fragilities and one polymer, could not confirm the proposed linear relationship between $m_{P}$ and $\alpha$, in agreement with earlier work by Buchenau et al. It was also suggested that a decomposition of the isobaric fragility into two terms, relating to the density and temperature contributions, should provide a more stringent test of this correlation, but no satisfactory correlation was found either in this case.

In the present, we focus on one of the simplest ways to tune fragility without changing the intermolecular interactions. This is achieved by studying the effect of chain length variation for two very different model polymers: polystyrene (PS) and polyisobutylene (PIB). These polymers exhibit an opposite change of fragility with molecular weight $M_{\mathrm{w}}:{ }^{14,24,25}$ while the fragility $m_{P}$ almost doubles with $M_{\mathrm{w}}$ for PS, it slightly decreases for PIB. This comparison should reveal whether there are indeed some intrinsic correlations between $m_{P}$ and $\alpha$. For this reason, a large set of inelastic X-ray scattering experiments were carried out on these two systems at various temperatures below $T_{\mathrm{g}}$. Our analysis reveals that the proposed correlation fails for both polymers. Although PS and PIB exhibit different molecular weight dependences of $\alpha$, none follows the expected behavior with fragility. We argue that the failure of such a correlation is not related to a secondary $\beta$ relaxation, as has been proposed recently in ref 26 . We speculate that many regularities known for small molecules fail in the case of polymers due to the polymer specific contribution to fragility (e.g., intramolecular degrees of freedom and energy barriers).

\section{Experimental Section}

The weight-averaged molecular weights $M_{\mathrm{w}}$ and polydispersity indices $I_{\mathrm{p}}$ of the selected samples, and their glass transition temperatures $T_{\mathrm{g}}$, obtained by differential scanning calorimetry
Table 1. Characteristics of the Samples

\begin{tabular}{lrrll}
\hline \multicolumn{1}{c}{ sample } & $\begin{array}{c}M_{\mathrm{w}} \\
\left(\mathrm{g} \cdot \mathrm{mol}^{-1}\right)\end{array}$ & \multicolumn{1}{c}{$I_{\mathrm{p}}$} & \multicolumn{1}{c}{ source } & $T_{\mathrm{g}} / \mathrm{K}$ \\
\hline PIB 680 & 680 & 1.06 & Polymer Standard Service & 190 \\
PIB 1190 & 1190 & 1.08 & University of Akron & 196 \\
PIB 3580 & 3580 & 1.23 & Polymer Standard Service & 201 \\
PIB 500k & 500000 & 2.5 & Sigma-Aldrich & 205 \\
PS 820 & 820 & 1.11 & Scientific Polymer Products & 283 \\
PS 5110 & 5110 & 1.07 & Scientific Polymer Products & 358 \\
PS 16k & 16600 & 1.05 & Scientific Polymer Products & 366 \\
PS 97k & 97400 & 1.01 & Scientific Polymer Products & 373.5
\end{tabular}

with a heating rate of $10 \mathrm{~K} \mathrm{~min}^{-1}$, are presented in Table 1 . The products were purchased from various companies as listed and used as received.

The IXS experiments were performed on ID28 at the ESRF in Grenoble, France. We used the $(11,11,11)$ reflection of the $\mathrm{Si}$ monochromator and analyzer crystals; the corresponding resolution is fwhm $=1.5 \mathrm{meV}{ }^{27}$ The integration times were comparable for all measurements ( $\sim 6 \mathrm{~h}$ per spectra). The lower molecular weight polystyrene sample was conditioned in a standard glass cell with diamond windows. The other PS samples of higher $T_{\mathrm{g}}$ were prepared in aluminum hollow cylinders opened at both ends (without any windows). A piece of tantalum was positioned at the end of the cells in order to reduce the background resulting mainly from the scattering of the Kapton windows of the cryostat. Hightemperature experiments were performed in a different sample environment using a furnace.

The PIB samples were measured during earlier high-pressure experiments in a pressure cell, including at atmospheric pressure. ${ }^{24} \mathrm{~A}$ hollow Teflon cylinder, closed at both ends by a Teflon film, was filled with the polymer. It was then placed in the pressure cell and surrounded by ethanol. The pressure cell was sealed at both ends by $1 \mathrm{~mm}$ thick diamond windows. The contribution to the scattering of the empty pressure cell, which is much higher than for standard cells, was measured in exactly the same conditions and subtracted from the obtained spectra.

The treatment of both data sets was performed using standard procedures by fitting the dynamic structure factor $S(Q, \omega)$ as a function of the energy $\omega$, using the sum of a delta function $\delta(\omega)$ and a damped harmonic oscillator (DHO) function convoluted with the resolution of the instrument: ${ }^{24,28,29}$

$$
\begin{aligned}
& S(Q, \omega)= \\
& \quad S(Q)\left(f(Q) \delta(\omega)+[1-f(Q)] \frac{1}{\pi} \frac{\Omega^{2} \Gamma(Q)}{\left(\omega^{2}-\Omega^{2}(Q)\right)^{2}+\omega^{2} \Gamma^{2}(Q)}\right)
\end{aligned}
$$

Here, $S(Q)$ is the static structure factor, $\Omega(Q)$ is the energy of the longitudinal acoustic-like modes, $\Gamma(Q)$ is its broadening, and $f_{Q}$ is the nonergodicity factor. This procedure gives a direct estimate of the nonergodicity factor for each spectrum at a given wave vector $Q$. The nonergodicity factors and $\alpha$ values presented in this paper were all obtained at a wave vector of $Q=2 \mathrm{~nm}^{-1}$, a good compromise between smaller $Q$ where the Brillouin peaks are difficult to resolve and higher $Q$ where the dynamical structure factor starts to be modulated by the static structure factor. ${ }^{30,31}$

\section{Choice of Sample}

PIB and PS exhibit opposite molecular weight dependences for many of their physical properties. The first important difference between the two polymers lies in the values of the glass transition temperatures and especially their sensitivity to the chain length. It is well-known that $T_{\mathrm{g}}$ always increases with chain length (for chains with nonfunctionalized ends). Nevertheless, the $T_{\mathrm{g}}$ increase varies from one polymer to another: it is very large for PS (more than $150 \mathrm{~K}$ ) but small for PIB (around $20 \mathrm{~K}$ ), as illustrated in Figure 1a. 

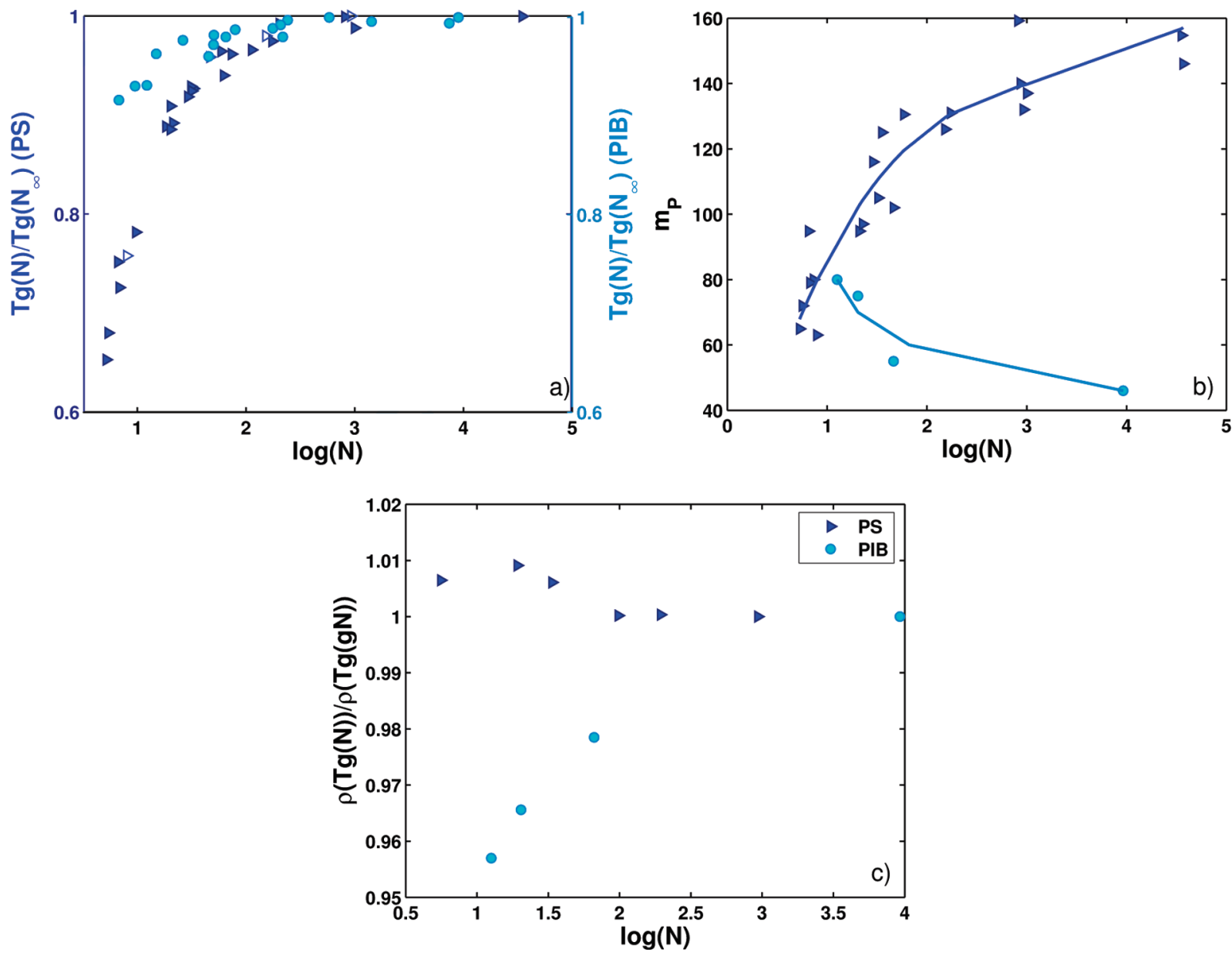

Figure 1. (a) Dependence of the glass transition temperature $T_{\mathrm{g}}$ on the number of monomers, $N$, normalized by the value for the longest chain $T_{\mathrm{g}}\left(N_{\infty}\right)$ of PS (left $y$ axis and open circles) and PIB (right $y$ axis and open triangles). Values from our DSC experiments and literature data. ${ }^{32-36}$ (b) $N$ dependence of the isobaric fragility $m_{P}$ for PS and PIB. The fragilities of the two intermediate molecular weights of PIB are deduced from other works (see text for details). The lines are guides to the eyes. (c) Density $\rho\left(T_{\mathrm{g}}\right)$ estimated at the glass transition temperature of each sample and normalized by $\rho\left(T_{\mathrm{g}}\left(N_{\infty}\right)\right)$ of the highest molecular weight sample for comparison. ${ }^{37}$

While the chain length dependence of $T_{\mathrm{g}}$ follows a general trend for all polymers, the dependence of the fragility is, on the contrary, less predictable. It increases for most polymers (sometimes by more than a factor of 2, as in the case for PS); in some cases it stays constant (e.g., in polydimethylsiloxane, PDMS) or displays a slight decrease (e.g., in PIB) with chain length. Figure $1 \mathrm{~b}$ represents the molecular weight dependence of the fragility for our systems.

Fragility increases from 60 to 160 with increasing molecular weight of PS. ${ }^{33,38}$ In the case of PIB, the fragility decreases slightly with molecular weight. This trend was recently confirmed by various experimental techniques. ${ }^{14}$ However, the absolute values of fragility and the amplitude of its change are very sensitive to the experimental technique used, to the time scale chosen for the definition of $T_{\mathrm{g}}$, and to the exact chemical nature of the chain ends. Accordingly, all the PIB data plotted in the figure correspond to measurements taken at the same relaxation time $\tau_{\alpha}=100 \mathrm{~s}$. In the cases of the highest and lowest molecular weight PIB samples, the measurements were carried out on the same samples as the ones used for the IXS experiments: the highest $M_{\mathrm{w}}$ sample was studied with dielectric spectroscopy, ${ }^{39}$ and the lowest $M_{\mathrm{w}}$ sample was studied by mechanical measurements. ${ }^{40}$ The fragilities of the two other PIB samples were estimated using calorimetric data from ref 14 . These estimates would correspond to $m_{P}$ measured at a relaxation time of $100 \mathrm{~s}$. We would like to emphasize here that, for our discussions, we focus only on the trends of the variation in fragility with chain length and not on the absolute values.
While it is well-known that the density at constant temperature above $T_{\mathrm{g}}$ increases with molecular weight, the density measured at $T_{\mathrm{g}}, \rho\left(T_{\mathrm{g}}(N)\right)$, might have different behavior due to the change of the glass transition temperature with molecular weight. It appears that $\rho\left(T_{\mathrm{g}}(N)\right)$ also shows opposite molecular weight dependences in PS and PIB (Figure 1c): the density at $T_{\mathrm{g}}$ of PS samples slightly decreases (within $1 \%$ ), while the density of PIB increases by almost 3\% with molecular weight. We emphasize that the $3 \%$ variation is significant when compared to the total variation of density of $\sim 5-10 \%$ observed upon cooling to $T_{\mathrm{g}}$ at constant atmospheric pressure. The opposite dependences of fragility and density in PS and PIB on chain length make them ideal systems to test correlations between fragility and any other property.

\section{Results and Analysis}

Figures 2 and 3 present the raw IXS spectra for PS and PIB with different molecular weights, measured at low temperatures and at the glass transition temperatures, respectively. The spectra of polyisobutylene exhibit clear variations in the inelastic contribution (Brillouin side lines) with molecular weight over the entire temperature range. This change corresponds to an almost $20 \%$ increase in the speed of sound (determined as $\Omega / Q$ obtained by fitting the results) with molecular weight and is consistent with earlier low-temperature $(100 \mathrm{~K})$ light scattering data (at a 2 orders of magnitude smaller wave vector). ${ }^{25}$ Almost no influence of 
a)
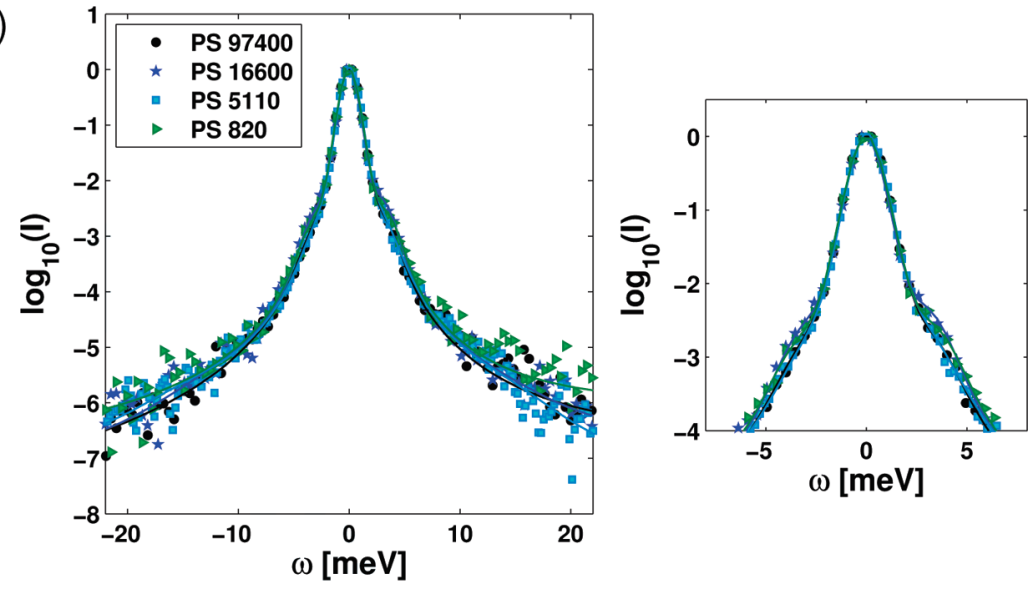

b)
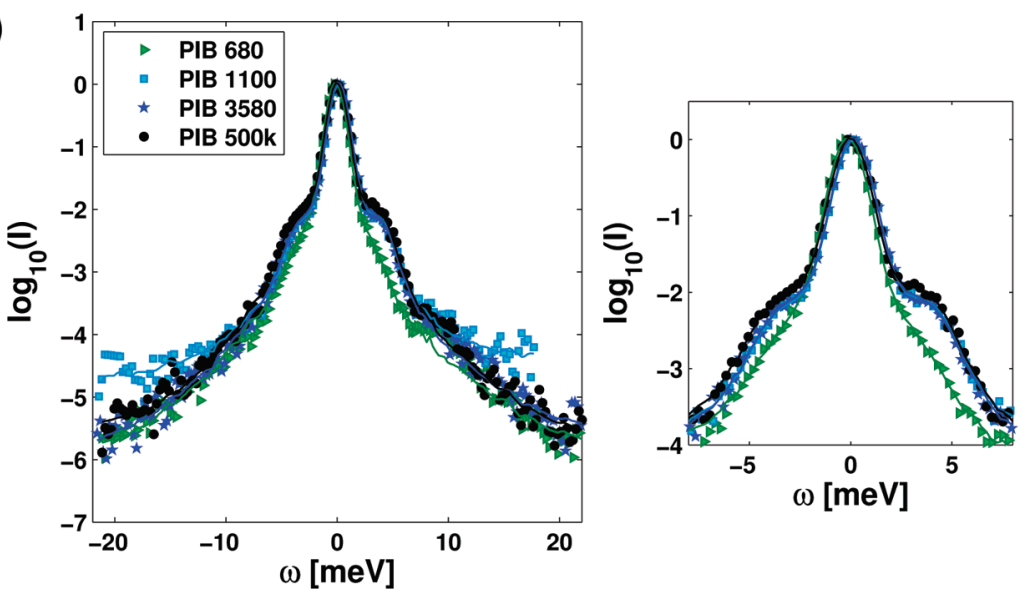

Figure 2. Inelastic X-ray scattering spectra measured at $2 \mathrm{~nm}^{-1}$ at low temperature in the glassy state. (a) Polystyrene of different molecular weights: PS820, PS5110, PS16600 and PS97400 at $80 \mathrm{~K}$, over a restricted range in $x$ on the right. (b) Polyisobutylene of different molecular weight: PIB680, PIB1 100, PIB3580, and PIB500k at $140 \mathrm{~K}$; similar results are found at lower temperature, but fewer samples were measured, over a restricted range in $x$ on the right. The symbols correspond to experimental data while the lines are fits to the data according to eq 2 .

molecular weight is observed for the PS spectra at $T_{\mathrm{g}}$. There is only a small difference between the spectra at lower temperature $(80 \mathrm{~K})$. These results are again consistent with the earlier light scattering data for PS at $80 \mathrm{~K},{ }^{25}$ where the speed of sound at low temperatures was found to decrease by less than $10 \%$ with increases in molecular weight.

From the fits of the IXS spectra, one can extract the nonergodicity parameter, $f_{Q}$, and the temperature dependence of its inverse value, $1 / f_{Q}$, in the glassy state. This provides an estimate of the $\alpha$ parameter as defined by Scopigno et al. in ref 22 . The temperature dependences of the inverse nonergodicity factor for PS and PIB are represented in Figure 4 versus the absolute temperature (a and c) and versus $T / T_{\mathrm{g}}$ (b and d). These plots show that the $\alpha$ parameter (defined by equation eq 1 ) increases with an increase in molecular weight for PIB, while it is almost independent of molecular weight (within experimental uncertainty) for the case of PS. We would like to emphasize that this effect does not come from the normalization by the glass transition temperature: the same trends are observed in plots of inverse nonergodicity factor versus absolute temperature.

Figure 5 displays the dependence of the $\alpha$ parameter on chain length for the two polymers. Similarly to the behavior of fragility and density at $T_{\mathrm{g}}$, a very different behavior is observed for the $\alpha$ parameter of the two systems. Moreover, a comparison of Figure 5 and Figure $1 \mathrm{~b}$ reveals a strong similarity between the chain length dependence of the density taken at $T_{\mathrm{g}}$ and of the $\alpha$ values.

\section{Discussion}

We now analyze a possible correlation between the $\alpha$ parameter and the fragility. Figure 6 presents the results for the $\alpha$ parameter plotted versus the isobaric fragility for the studied polymers including data points from the literature. The linear relationship found by Scopigno et al. is shown by the black line. From our results, it is clear that the correlation between $\alpha$ and $m_{P}$ fails for the two polymeric systems of different molecular weights, and we will discuss possible reasons for the failure of this correlation.

Figure 6 also presents literature data ${ }^{23}$ for high molecular weight PIB and PS that disagree with our present work. These discrepancies could be due to the way in which the different $\alpha$ values have been obtained. In the case of PIB, $\alpha$ was estimated in ref 23 from the isothermal compressibility. The latter was calculated from the static structure factor, $S(Q)$, measured by small-angle neutron scattering, ${ }^{41}$ and extrapolated to $Q=0$. The SANS data were measured at very high temperatures $(355 \mathrm{~K}$ or higher), i.e., well above the glass transition temperature, in a regime where the temperature dependence of the nonergodicity factor follows trends other than those discussed here (it tends to be less steep). For this reason, calculation of $\alpha$ based on the nonergodicity factor at $T \gg T_{\mathrm{g}}$ underestimates its value. Moreover, multiple scattering contribution that may still be present in the neutron data would also contribute to an underestimation of $\alpha$ resulting from an overestimation of $S(Q \rightarrow 0)$ compared to IXS experiment. 
a)

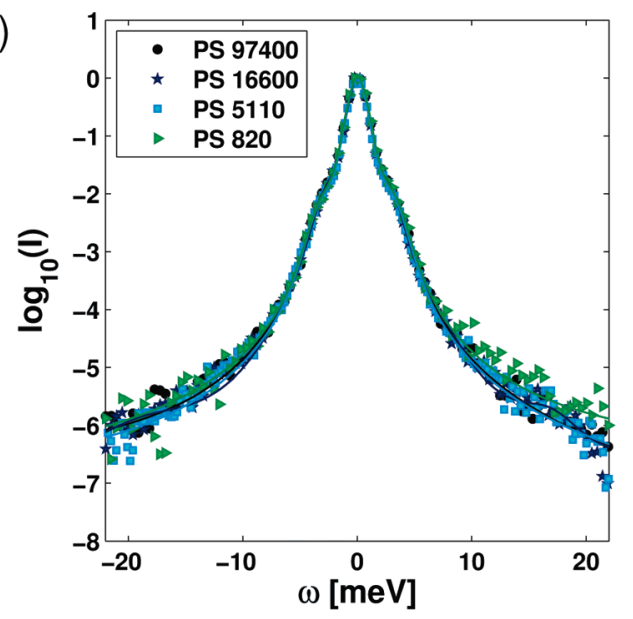

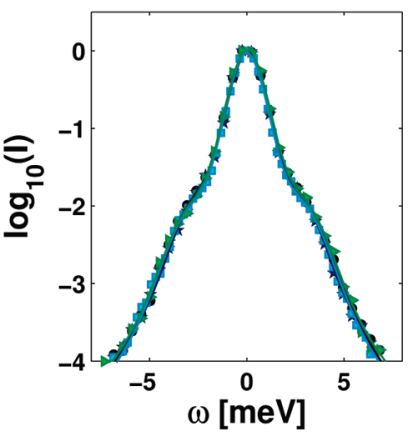

b)
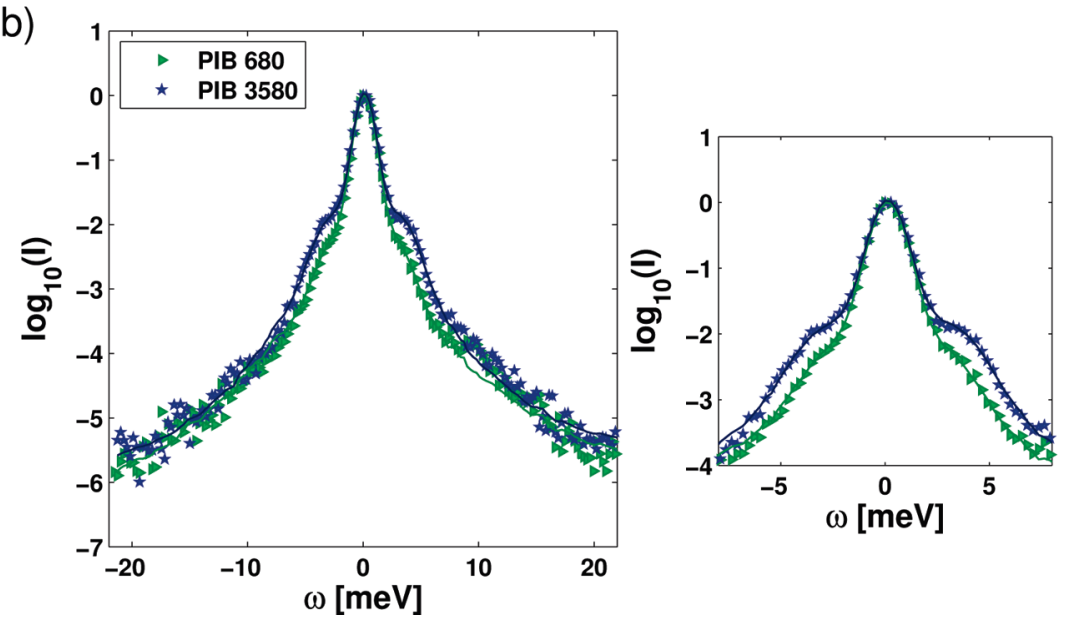

Figure 3. Inelastic X-ray scattering spectra measured at $2 \mathrm{~nm}^{-1}$ at the respective (molecular weight dependent) glass transition temperatures of each sample. (a) Polystyrene of different molecular weights: PS820, PS5110, PS16600, and PS97400 at $80 \mathrm{~K}$, over a restricted range in $x$ on the right. (b) Polyisobutylene of different molecular weight: PIB680 and PIB3580, over a restricted range in $x$ on the right. The symbols correspond to experimental data while the lines are fits to the data according to eq 2.

In the case of PS, the $\alpha$ value given by Buchenau et al. ${ }^{23}$ is calculated from compressibility values, estimated from the speed of sound in the sample, and is higher than our value. Our estimate of the speed of sound at low temperatures in PS from the IXS data (around $2750 \mathrm{~m} \cdot \mathrm{s}^{-1}$ ) agrees well with earlier low-temperature light scattering data on the same sample, while the value used in ref 23, i.e., $2219 \mathrm{~m} \cdot \mathrm{s}^{-1}$, seems rather low. Moreover, using the same approach outlined in ref 23 , we can estimate the $\alpha$ parameter from our value for the speed of sound $V_{l}$, using the equation

$$
\alpha_{\chi}\left(T_{\mathrm{g}}\right)=\frac{\chi_{\text {Brill }}\left(T_{\mathrm{g}}\right)}{\chi_{T}\left(T_{\mathrm{g}}\right)-\chi_{\text {Brill }}\left(T_{\mathrm{g}}\right)}
$$

with $\chi_{\text {Brill }}=1 / \rho V_{l}^{2}$. The value of $\alpha$ obtained from this approach agrees well with the value estimated from the slope of $f_{Q}$. Thus, the difference observed for the PS might be explained by the low value of the speed of sound used in ref 23. This low value is due to the chosen high temperature at $T=T_{\mathrm{g}} \sim 375 \mathrm{~K}$ and leads to overestimate the $\alpha$ parameter.

In any case, our results clearly demonstrate a failure of the suggested correlations between $\alpha$ and $m_{P}$. We must emphasize that our studies involve variations of fragility without chemical modification of the studied samples, just by an increase in the length of the polymer chains. Even if the two studied systems, PS and PIB, behave very differently, neither agrees with the earlier proposed correlation. This failure of the correlation for the two polymers can be related either to the definition of $f_{Q}$ used or the fragility itself. $f_{Q}$ might include contributions from several relaxation processes which are not related to the structural relaxation and thus the fragility. The fragility may be more complex for polymers than for some other glass-formers and might include some "polymer specific" contributions; ${ }^{14,42}$ the applicability of the fragility concept to polymers itself was recently questioned in ref 43 .

Recently, it has been proposed ${ }^{26}$ that a secondary (e.g., $\beta$ ) relaxation might strongly influence the measured values of the $\alpha$ parameter. The absolute values of $f_{Q}$ can indeed be affected not only by the structural relaxation but also by any other relaxation process entering the spectral window which would lead to a smaller $f_{Q}$. In the case of PS, however, mechanical relaxation and dielectric spectroscopy data have shown that the $\beta$ relaxation is similar for all molecular weights. ${ }^{38,44}$ In other words, even if the $\alpha$ parameter is affected by the $\beta$ relaxation, the effect would be the same for all molecular weights, consistent with a constant $f_{Q}$ for PS samples of different molecular weights. However, the fragility of PS increases strongly with molecular weight, but this is not reflected in the parameter $\alpha$. PIB shows very weak $\beta$ relaxation $^{16}$ in dielectric spectroscopy, and we therefore do not expect it to have an impact on the $\alpha$ values. Yet the $\alpha$ parameter changes significantly with $M_{\mathrm{w}}$ in PIB, while fragility shows only weak variations. Thus, the proposed explanation based on the role of secondary relaxation(s) cannot consistently describe all the data.

A separate question is whether the $Q$ range of our studies can still be considered to be in the long wavelength regime. In the case 

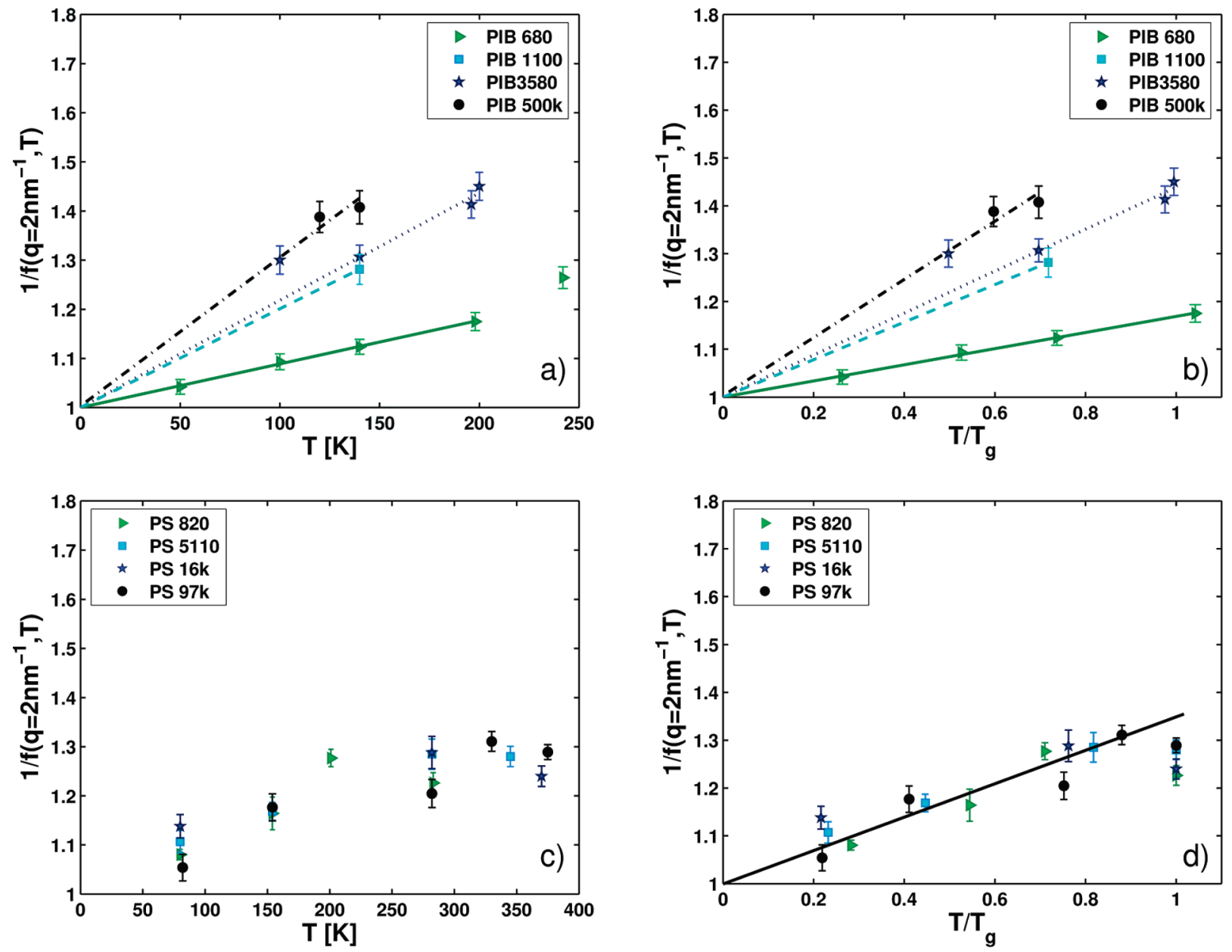

Figure 4. Inverse nonergodicity factor versus temperature (a and c) and temperature scaled by the respective glass transition temperatures of each sample, $T / T_{\mathrm{g}}$ (b and d), for different molecular weights of polyisobutylene (upper) and polystyrene (lower). The fits include the point of zero temperature, where $f_{Q}$ is equal to one by definition, and exclude the points at $T_{\mathrm{g}}$ and higher temperatures where the data already start to bend over as reported in ref 24. Because of very small values of the inverse nonergodicity factor and uncertainties, no large differences can be seen for PS between the plots against $T$ and $T / T_{\mathrm{g}}$, even considering the very large change in $T_{\mathrm{g}}$ with molecular weight.

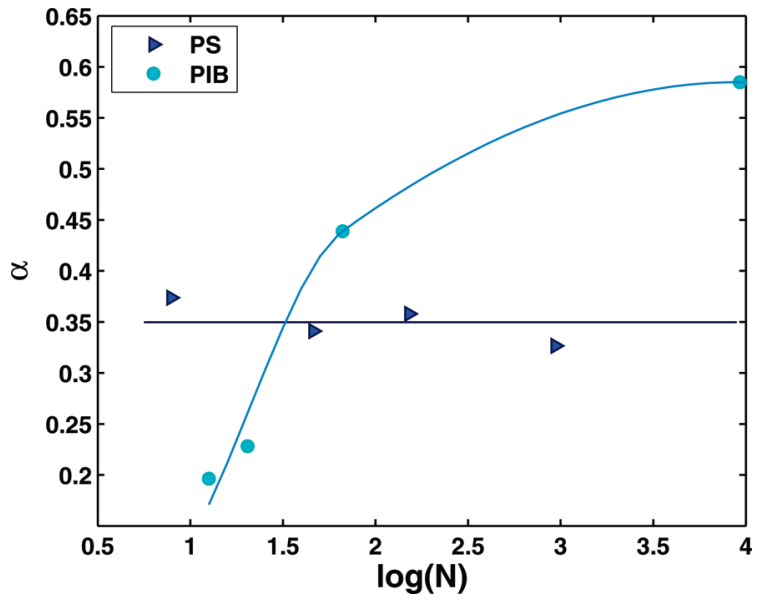

Figure 5. $\alpha$ parameter versus chain length $N$ for PS and PIB, extracted from the previous figures at low temperature. Lines are guides for the eyes.

of PIB, $S(Q)$ displays its main peak around $11 \mathrm{~nm}^{-1},{ }^{41}$ which is quite far from the $Q$ value used in our measurements, $2 \mathrm{~nm}^{-1}$. In PS, $S(Q)$ shows two peaks in this wave vector range, ${ }^{31}$ the first appearing around $5 \mathrm{~nm}^{-1}$. This peak corresponds to the interchain distance and changes slightly with molecular weight. The second peak, associated with phenyl rings, appears around $13 \mathrm{~nm}^{-1}$

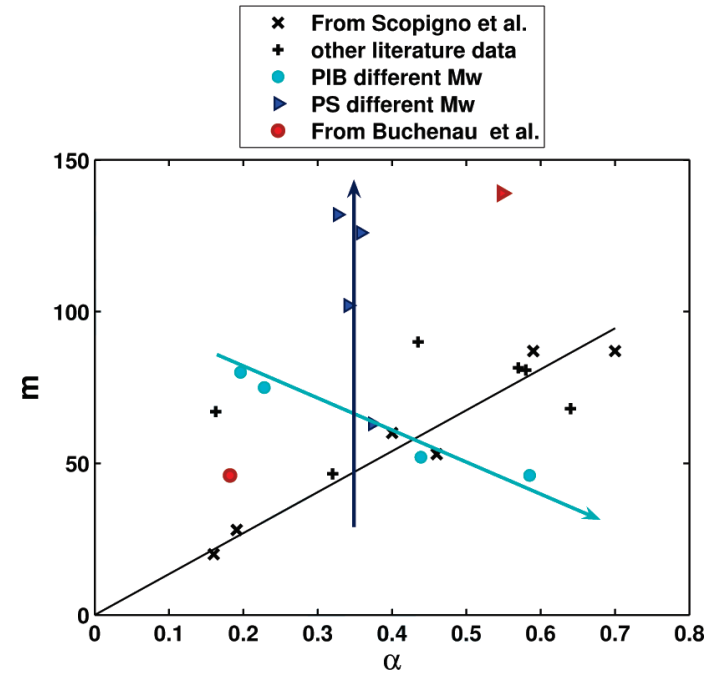

Figure 6. The $\alpha$ parameter versus the isobaric fragility $m_{P}$ for different molecular liquids and for the PIB and PS samples at different molecular weights. Crosses represent $\alpha$ calculated from IXS data in other studies. Red symbols for polystyrene (triangle) and polyisobutylene (circle) correspond to $\alpha$ values calculated from the compressibilities of high molecular weight polymers from ref 23 . The arrows indicate the direction from shorter chains to longer chains. The straight lines shows the result of the relationship $\alpha=(135 \pm 10) m_{P}$ from ref 22 . 
and is independent of molecular weight. Thus, the $Q$ range chosen for $f_{Q}$ may not be so far from the peak in $S(Q)$ in the case of PS. However, the good agreement between the speed of sound estimated at the wave vector $Q$ of IXS and that estimated by light scattering suggests that the IXS data are not affected by the close proximity to the diffraction peak.

Let us now consider the other parameter of interest: fragility. We already stressed in ref 24 that the choice of the isobaric fragility (calculated at constant pressure) should be addressed. Hence, the first question is whether the isochoric fragility $m_{\rho}{ }^{11,45}$ (calculated at constant volume) should rather be used to try to correlate with $\alpha$, as it has been previously introduced. ${ }^{46}$ For PS, the variation of $m_{\rho}$ with molecular weight was estimated by combining thermodynamic data and ratios from ref 47 . It changes by $40 \%$, from 62 to 105 for PS 13000 to $90000 \mathrm{~g} \mathrm{~mol}^{-1}$, while the change in $m_{P}$ was only $25 \%$, from 120 to 160 over the same molecular weight range. The correlation with $m_{\rho}$ would therefore be even worse than that with $m_{P}$ in these cases. Thus, decomposing fragility in terms of temperature and density contribution does not seem to affect our conclusions.

The impact of the connectivity of the polymer chain on the dynamics and on the fragility is important ${ }^{14,43,48}$ and may explain the usually much higher fragility of polymers when compared to molecular liquids. However, many other parameters that usually correlate with fragility in small molecules (e.g., the $\alpha$ parameter) are less affected by the connectivity, at least for PS. We emphasize that a similar breakdown of correlations in the case of PS is known for (i) correlations between fragility and the ratio of the bulk to shear modulus, ${ }^{19}$ (ii) correlations between the jump in specific heat at $T_{\mathrm{g}}$ and fragility, ${ }^{49,50}$ and (iii) breakdown of the Adams-Gibbs relation between thermodynamic measurements and the temperature variations of structural relaxation times. ${ }^{51}$ All these results suggest that there may indeed be some "polymer specific" contributions to fragility that does not show up in other properties (e.g., the nonergodicity parameter, Poisson ratio, jump in specific heat at $T_{\mathrm{g}}$, etc.). This contribution to fragility remains unknown and is most probably related to intramolecular degrees of freedom, contribution of intramolecular energy barriers, or some other polymer specific properties.

\section{Conclusions}

With the aim to test possible correlations between properties of the glass (taken as a snapshot of the liquid, frozen at $T_{\mathrm{g}}$ ) and properties of the liquid such as fragility, we have performed IXS experiments on polymers with different chain lengths. We specifically chose PS and PIB because these polymers exhibit opposite behaviors of many parameters (e.g., fragility, density at $T_{\mathrm{g}}$ ) with molecular weight. Our results clearly demonstrate that the earlier proposed correlation between fragility and the $\alpha$ parameter does not hold in these systems. PS and PIB exhibit very different molecular weight dependence of the $\alpha$ parameter, but none shows the suggested correlation to fragility. We emphasize that this failure cannot be explained by the existence of the $\beta$-relaxation because the latter is essentially molecular weight independent for PS and is extremely weak in PIB.

It seems that the attempt to generalize the "fragilitynonergodicity" relationship from molecular liquids to polymers might be conceptually incorrect. In our opinion, this is because the chain connectivity and contributions of intramolecular degrees of freedom add extra contributions to the fragility of polymers. Therefore, it might even be that any correlation of properties of the glass with the fragility established for small molecules might not be applicable to polymers.

This underlines once more the importance of the chain connectivity on the structural relaxation in polymeric systems, as had been discussed recently. ${ }^{48}$ From our study it becomes also obvious that the ingredients to fragility have to be understood better in general and in particular for polymers.

Acknowledgment. A. Sokolov acknowledges financial support by the NSF Polymer program (Grant DMR-0804571). This work was supported by the CNRS and CEA (France). The authors thank the ESRF for the beam time.

\section{References and Notes}

(1) Angell, C. A. J. Non-Cryst. Solids 1991, 131, 13-31.

(2) Adam, G.; Gibbs, J. H. J. Chem. Phys. 1965, 43, 139-146.

(3) Cohen, M. H.; Turnbull, D. J. Chem. Phys. 1959, 31, 1164-1169.

(4) Goldstein, M. Faraday Symp. Chem. Soc. 1972, 6, 7-13.

(5) Bouchaud, J. P. J. Phys. I 1992, 2, 1705-1713.

(6) Dyre, J. C. Phys. Rev. Lett. 1987, 58, 792-795.

(7) Götze, W.; Sjögren, L. Rep. Prog. Phys. 1992, 55, 241-376.

(8) Debenedetti, P. G.; Stillinger, F. Nature 2001, 410, 259-267.

(9) Xia, X.; Wolynes, P. Proc. Natl. Acad. Sci. U.S.A. 2000, 97, 29902994.

(10) Schweizer, K.; Saltzman, E. J. Chem. Phys. 2003, 119, 1181.

(11) Niss, K.; Alba-Simionesco, C. Phys. Rev. B 2006, 74, 024205.

(12) Niss, K.; Dalle-Ferrier, C.; Tarjus, G.; Alba-Simionesco, C. J. Phys.: Condens. Matter 2007, 19, 076102.

(13) Frick, B.; Dosseh, G.; Cailliaux, A.; Alba-Simionesco, C. Chem. Phys. 2003, 292, 311-323.

(14) Sokolov, A.; Novikov, V.; Ding, Y. J. Phys.: Condens. Matter 2007, 19, 205116.

(15) Qin, Q.; McKenna, G. J. Non-Cryst. Solids 2006, 352, 2977-2985.

(16) Kunal, K.; Paluch, M.; Roland, C.; Puskas, J.; Chen, Y.; Sokolov, A. J. Polym. Sci., Part B 2008, 46, 1390-1399.

(17) Sokolov, A. P.; Rössler, E.; Kisliuk, A.; Quitmann, D. Phys. Rev. Lett. 1993, 71, 2062-2065.

(18) Ngai, K. L. J. Non-Cryst. Solids 2000, 275, 7-51.

(19) Novikov, V. N.; Ding, Y.; Sokolov, A. P. Phys. Rev. E 2005, 71, 061501

(20) Bove, L.; Petrillo, C.; Fontana, A.; Ivanov, A.; Dreyfus, C.; Sokolov, A. Physica B 2006, 385-386, 16.

(21) Dyre, J. Rev. Mod. Phys. 2006, 78, 953.

(22) Scopigno, T.; Ruocco, G.; Sette, F.; Monaco, G. Science 2003, 302, 849-852.

(23) Buchenau, U.; Wischnewski, A. Phys. Rev. B 2004, 70, 092201.

(24) Niss, K.; Dalle-Ferrier, C.; Giordano, V. M.; Monaco, G.; Frick, B.; Alba-Simionesco, C. J. Chem. Phys. 2008, 129, 194513.

(25) Ding, Y. F.; Novikov, V. N.; Sokolov, A. P.; Cailliaux, A.; Dalle-ferrier, C.; Alba-Simionesco, C.; Frick, B. Macromolecules 2004, 37, 9264-9272.

(26) Scopigno, T.; Cangialosi, D.; Ruocco, G. Phys. Rev. B 2010, 81, 100202.

(27) www.esrf.eu/UsersAndScience/Experiments/HRRS/ID28.

(28) Ruocco, G.; Sette, F. J. Phys.: Condens. Matter 2001, 13, 91419164.

(29) Monaco, G.; Giordano, V. Proc. Natl. Acad. Sci. U.S.A. 2009, 106, $3659-3663$.

(30) Iradi, I.; Alvarez, F.; Colmenero, J.; Arbe, A. Physica B 2004, 350, 881-884.

(31) Dalle-Ferrier, C.; Simon, S.; Zheng, W.; Badrinarayanan, P.; Fennell, T.; Frick, B.; Zanotti, J.; Alba-Simionesco, C. Phys. Rev. Lett. 2009, 103, 185702.

(32) Alberty, K. A.; Hogen-Esch, T. E.; Carlotti, S. Macromol. Chem. Phys. 2005, 206, 1035-1042.

(33) Roland, C. M.; Casalini, R. J. Chem. Phys. 2003, 119, 1838-1842.

(34) Rique-Lurbet, L.; Schappacher, M.; Deffieux, A. Macromolecules 1994, 27, 6318-6324.

(35) Santangelo, P. G.; Roland, C. M.; Chang, T.; Cho, D.; Roovers, J. Macromolecules 2001, 34 (26), 9002-9005.

(36) Robertson, C.; Roland, C. J. Polym. Sci., Part B 2004, 42, 2604 2611.

(37) The PS data were obtained from ref 52 and are consistent with data from ref 53 measured down to the glass. In the case of PIB, no density measurements have been systematically performed in a temperature range down to the glass. However, the thermal expansivity has been measured by isothermal calorimetric experiments, and the room temperature densities have been extrapolated from these values to the glass temperature. ${ }^{54}$ In the case of high molecular weight samples a large set of data measured in the liquid has been published ${ }^{33,55-57}$ displaying compatibility with each other 
(we used an average value here). In the case of the lower molecular weight sample, the only density data available are from ref 53 (these have been extrapolated to $T_{\mathrm{g}}$ using the following thermal expansivity $\alpha_{P}=6.6978 .10^{-4} \mathrm{~K}^{-1}$, measured at $210 \mathrm{~K}$ by isothermal calorimetry). For the two intermediate molecular weights, no experimental data were found; the densities have been extracted from MD simulations ${ }^{58}$ performed at $300 \mathrm{~K}, 1$ atm and extrapolated to $T_{\mathrm{g}}$.

(38) Hintermeyer, J.; Herrmann, A.; Kahlau, R.; Goiceanu, C.; Rössler, E. Macromolecules 2008, 41, 9335.9344.

(39) Arbe, A.; Colmenero, J.; Frick, B.; Monkenbusch, M.; Richter, D. Macromolecules 1998, 31, 1133 .

(40) Olsen, B. Private discussions.

(41) Farago, B.; Arbe, A.; Colmenero, J.; Faust, R.; Buchenau, U.; Richter, D. Phys. Rev. E 2002, 65, 051803.

(42) Kunal, K.; Robertson, C.; Pawlus, S.; Hahn, S. F.; Sokolov, A. P. Macromolecules 2008, 41, 7232-7238.

(43) Abou Elfadl, A.; Hermann, A.; Hintermeyer, J.; Petzold, N.; Novikov, V.; Roessler, E. Macromolecules 2009, 42, 6816-6817.

(44) Santangelo, P.; Roland, C. Macromolecules 1998, 31, 45814585 .
(45) Alba-Simionesco, C.; Tarjus, G. J. Non-Cryst. Solids 2006, 352, 4888-4894.

(46) Alba-Simionesco, C.; Cailliaux, A.; Alegria, A.; Tarjus, G. Europhys. Lett. 2004, 68, 58-64.

(47) Roland, C.; Mcgrath, K.; Casalini, R. J. Non-Cryst. Solids 2006, $352,4910$.

(48) Sokolov, A.; Schweizer, K. Phys. Rev. Lett. 2009, 102, 248301.

(49) Santangelo, P.; Roland, C. Phys. Rev. B 1998, 58, 14121-14123.

(50) Huang, D. H.; Mckenna, G. B. J. Chem. Phys. 2001, 114, 5621-5630.

(51) Cangialosi, D.; Alegria, A.; Colmenero, J. J. Chem. Phys. 2006, 124, 024906.

(52) Gray, R.; Harrison, G.; Lamb, J. Proc. R. Soc. London, A 1977, $356,77$.

(53) Zoller, P.; Walsh, D. Standard Pressure-Volume-Temperature Data for Polymers; Technomic Publishing Co.: Lancaster, PA, 1995.

(54) Chauty-Cailliaux, A. Ph.D. Thesis, Université de Paris XI, 2003.

(55) Eichinger, B.; Flory, P. Macromolecules 1968, 1, 285-286.

(56) Kilburn, D.; Wawryszczuk, J.; Dlubek, G.; Pionteck, J.; Hässler, R.; Alam, M. Macromol. Chem. Phys. 2006, 207, 721-734.

(57) Sanchez, I. C.; Cho, J. Polymer 1995, 36, 2929-2939.

(58) Rousseau, B. Private communication. 